\title{
Health Insurance Portability and Accountability Act is here: What price privacy?
}

\author{
Lynn D. Fleisher, PhD, JD $D^{1}$ and Laura J. Cole, $J D^{2}$
}

The Health Insurance Portability and Accountability Act of 1996 (HIPAA) created new requirements for health care providers to protect the privacy and security of health information. Pursuant to HIPAA, on November 3, 1999, the Secretary of the Department of Health and Human Services (HHS) proposed lengthy and detailed regulations, designed to protect the privacy of individually identifiable health information. For the next 13 months, HHS received and reviewed nearly 52,000 comments relating to the proposed regulations. On December 28, 2000, HHS published the regulations in final form. Amid much speculation that new HHS Secretary Tommy Thompson would delay the controversial rules, on April 12, 2001, President Bush ordered their immediate implementation. The HIPAA privacy rules (the "Privacy Rules") became effective on April 14, 2001; all health care providers must be in compliance with the Rules by April 14, 2003. This article provides a summary of the Privacy Rules requirements that are likely to impact the practice of medical genetics.

\section{GENERAL REQUIREMENTS}

The HIPAA Privacy Rules will require medical geneticists to take steps to protect the privacy of patients' and research subjects' personal health information. Under HIPAA, a geneticist who is a "covered entity" may not use or disclose an individual's protected health information unless permitted or required by the Privacy Rules. The term "covered entity" includes physicians, counselors, researchers, laboratories, hospitals, and other health care providers that transmit health information electronically in connection with certain administrative or billing transactions-i.e., essentially any health care provider who bills electronically. Such providers must establish privacy protections for all protected health information, whether contained in electronic or paper records or in oral communications.

\footnotetext{
The information in this article is provided as an educational resource for geneticists to make them aware of relevant new developments in the law. It is not intended as, and should not be considered, legal advice. Adherence to any information or suggestions contained in the article is strictly voluntary and does not ensure compliance with legal requirements, and the authors of the article and the editors and publishers of Genetics in Medicine expressly disclaim any responsibility or liability therefor. If you have specific questions regarding the information discussed in the article, please seek the advice of your legal counsel.

${ }^{I}$ Of Counsel to the law firm of Sidley Austin Brown \& Wood, Chicago, Illinois, and legal counsel to the American College of Medical Genetics, the American Board of Medical Genetics, and the American Board of Genetic Counseling; ${ }^{2}$ Associate in Sidley Austin Brown \& Wood's Health Care Group.

Lynn D. Fleisher, PhD, JD, Sidley Austin Brown \& Wood, Bank One Plaza, 10 South Dearborn, Chicago, IL 60603; E-mail: Ifleisher@sidley.com.
}

HIPAA protects all "individually identifiable health information." The term "individually identifiable health information" encompasses all information that (1) identifies an individual, or reasonably could be used to identify the individual (including deceased individuals); (2) is created or received by a health care provider; and (3) relates to the past, present, or future physical or mental health of the individual, the provision of health care to the individual, or the payment for the provision of health care to the individual.

In short, any medical geneticist who electronically transmits any individually identifiable information relating to the health of any patient or research subject will be subject to HIPAA. Once subject, the geneticist must comply with HIPAA for all protected health information (PHI), regardless of whether that particular information is transmitted electronically.

The key requirements of the Privacy Rules relate to (1) controlling the use and disclosure of PHI; (2) safeguarding patient rights; and (3) establishing, documenting, and implementing compliance policies and procedures. Ultimately, all geneticists, including physicians, genetic counselors, and laboratory scientists, will need to be reeducated and will be held to a new standard of privacy protection.

\section{USE AND DISCLOSURE OF PHI}

Under HIPAA, a medical geneticist generally must (1) obtain consent for the use or disclosure of PHI for treatment, payment, or health care operations purposes; (2) obtain authorization for the use or disclosure of PHI for other purposes, including research; (3) use or disclose only the minimum necessary PHI; and (4) ensure compliance by third parties to whom the geneticist discloses PHI.

\section{Consent}

HIPAA requires any health care provider who has a direct treatment relationship with a patient to obtain a valid consent to use or disclose the patient's PHI for treatment, payment, or health care operations purposes. The term "health care operations" is quite broad and not well-defined. However, it includes quality assessment and improvement activities; certain licensing and credentialing activities; and general business planning, management, and administrative activities. The HIPAA consent requirement does not, however, apply to providers such as laboratories who have only an "indirect treatment relationship" with patients. An indirect treatment relationship exists when the laboratory delivers health care to a patient based on orders from an- 
other provider and reports the results back to the other provider rather than directly to the patient.

Thus, under the Privacy Rules, a clinical geneticist who has a direct treatment relationship with patients will need to obtain a written consent from the patient or the patient's legal representative prior to using or disclosing the patient's PHI for any of these basic health care purposes. Although the consent may be fairly general, the Privacy Rules do establish certain requirements for form and content. A laboratory geneticist, on the other hand, because of the indirect treatment relationship, is likely to be exempt from the consent requirement and may use or disclose PHI for treatment, payment, or health care operations purposes without obtaining consent from the patient.

Importantly, a clinical geneticist who is employed by a hospital or other covered entity, or is otherwise part of an "organized health care arrangement," may be included in a "joint consent." The joint consent must identify with reasonable specificity at least the "class" of providers that are covered-e.g., physicians, genetic counselors. Since the Privacy Rules do not require hospitals to include individual providers on their consent forms, clinical geneticists should ensure that (1) they are covered by the hospital's consent form, (2) they are comfortable that the consent meets the regulatory requirements, and (3) the hospital will advise them if the consent is revoked. If a joint consent is used, a joint privacy notice (discussed below) also must be used. Also, if and when the geneticist acts outside of the scope of hospital practice, or sees patients who have not signed an appropriate hospital consent, the geneticist will need to obtain consent from those patients.

\section{Authorization}

In addition to the consent requirement, the Privacy Rules require health care providers to obtain a more specific form of permission, a written "authorization," prior to using or disclosing a patient's PHI for purposes other than treatment, payment, or health care operations, including for research purposes. The authorization requirements apply to all providers, both direct and indirect. The Privacy Rules establish many specific requirements for the form and content of the authorization. The overriding concern is that an authorization must provide sufficient information to allow the individual to make an informed decision whether to authorize the use or disclosure of PHI.

\section{Requirements for authorization}

An authorization must include (1) a description of the PHI to be used or disclosed; (2) a list or description of who may use or disclose the information; and (3) a list or description of who the intended recipients of the information will be. It also must include an expiration date or event, and statements informing the individual that (1) he or she may, with certain exceptions, revoke the authorization, and (2) PHI used or disclosed may be subject to redisclosure and no longer protected by the Privacy Rules.

In some cases, the purpose(s) for which the information will be used or disclosed must be included. (Even where it is not required by HIPAA, the purpose of the research may be a necessary element of informed consent under state law.) The information provided on the authorization form must be specific and clear; it cannot simply be a general description such as "for research." It also must be written in plain English, not medical jargon, and must be current and valid at the time of the use or disclosure.

For example, the description of the information to be used or disclosed may be explained as "all results of genetic tests performed in July 2000," or "results of genetic tests performed by Dr. Mendel," or "the patient's entire medical record." The description of who may use or disclose the information should indicate a specific person or people, or a specific class of people. For example, the authorization may state that the information may be disclosed by Dr. Mendel, or that it may be used by all geneticists at a certain hospital, or that it may be used by all geneticists, genetic counselors, nurses, and other medical practitioners at a specific hospital. Similarly, the description of who may receive the information should list all expected recipients or classes of expected recipients. For example, the authorization may state the information may be disclosed to hospital administrators, organizations providing grant funds, academic journals, and pharmaceutical companies that are interested in manufacturing drugs to use in treating the disease.

\section{Exceptions to authorization requirement}

Although the authorization requirement is quite broad, HIPAA establishes some fairly substantial exceptions that lessen the burden. First, a provider does not need a patient's authorization to disclose PHI to the patient's family or friends-if such disclosure is necessary to enable those persons to assist in the patient's care. The patient must have the opportunity to object prior to such disclosure, but need not sign a written authorization. In the case of an unemancipated minor, a parent or guardian generally will be considered to be the child's legal representative for purposes of receiving the child's PHI, as well as for providing any necessary consent or authorization on behalf of the minor.

Thus, for example, if a patient were scheduled for BRCA 1 testing, the geneticist may inform a relative who will drive the patient to the testing facility of the time and place of the appointment and any instructions that may be relevant to prepare for the test or to care for the patient thereafter. The geneticist may not, however, disclose the purpose of the screening, i.e., that a BRCA 1 test-or even a "genetic" test—will be performed, unless (1) such information is necessary for the relative to appropriately assist the patient, or (2) the patient has specifically authorized the disclosure. Of particular importance to geneticists is that they may disclose PHI to a family member only to the extent that the family member needs the information to assist in the patient's care-not to inform, warn, or otherwise benefit the family member.

It is also important to note that the permission provided by HIPAA to disclose a patient's PHI to a family or friend, as well as for the other authorizable uses and disclosures, does not free the geneticist from other ethical and legal obligations to determine the appropriateness of disclosing a patient's health information. Rather, when the geneticist determines that it is appropriate to disclose such information, HIPAA determines whether the geneticist may do so without obtaining separate written authorization. 
HIPAA also permits a health care provider to disclose a patient's PHI for certain public health and health oversight activities. For example, a geneticist may disclose PHI in an adverse event report to the Food and Drug Administration; in the course of an investigation, or a disciplinary, judicial, or administrative proceeding; in response to a proper subpoena or discovery request; to assist a coroner in determining the cause of a death; or to facilitate organ transplantation.

A geneticist also may disclose limited PHI to law enforcement officials to assist in locating a suspect, fugitive, material witness, or missing person. Such information may include an individual's $\mathrm{ABO}$ blood type, rh factor, and a description of physical characteristics, such as height, weight, race, hair color, and eye color. Disclosure for law enforcement purposes may not, however, include PHI related to an individual's DNA or to typing, samples, or analysis of the individual's body fluids or tissue for such purposes.

\section{Minimum necessary information}

All uses and disclosures of PHI should be narrowly tailored to provide maximum privacy protection. The Privacy Rules require that health care providers comply with a "minimum necessary standard." Under that standard, except in certain situations, a provider must make reasonable efforts to limit uses and disclosures of PHI to the minimum information necessary to accomplish the purpose of the use or disclosure. Thus, for example, even if a consent or an authorization would permit a geneticist to disclose a patient's entire medical record, if the purpose of the disclosure can be accomplished by disclosing just a portion of that record, the geneticist may not disclose more than that portion of the record. The minimum necessary requirement does not apply, however, to uses and disclosures to another health care provider for treatment purposes.

\section{Business associate agreements}

Health care providers also must ensure that any third party to whom the provider discloses PHI agrees to protect the information. Unless an exception applies, a provider must enter into a written "business associate agreement" with each third party that performs services on behalf of the provider and that has access to PHI. A business associate agreement must meet certain HIPAA requirements regarding form and content. In general, the agreement must require the business associate to treat the $\mathrm{PHI}$ in the same way that the provider must treat such information. For example, a geneticist may have to enter into a business associate agreement with his or her accountant, attorney, billing company, consultants, or similar other third parties if they will have access to patients' PHI. Like the consent requirement, however, the business associate agreement requirement likely will be met by the inclusion of individual geneticists in a joint agreement executed between a hospital or other health care entity and its organizational business associates.

\section{De-identification}

PHI will not be subject to the Privacy Rules if it has been totally stripped of all personal identifiers. PHI is considered "de-identified" if it does not identify an individual and there is no reasonable basis to believe that it can be used to identify an individual. The Privacy Rules provide two different ways for a geneticist to de-identify PHI. The first option is to obtain a determination by a person with appropriate statistical and scientific knowledge and experience that the risk of re-identification is very small. For most geneticists, however, obtaining this type of determination on a regular basis will not be practicable.

Alternatively, PHI will be considered de-identified if it meets a type of "safe harbor" provided in the Privacy Rules; that is, it must be stripped of all 18 identifiers enumerated in the Privacy Rules. The identifiers required to be deleted include standard types of identifiers, such as name, address, telephone number, fax number, email address, social security number, medical record number, and health plan membership number. In addition, de-identified information may not include any dates relating to the individual that are more specific than years, with ages 90 and over aggregated into a single category of " 90 and older"; any account number, certificate number, license number, or any other traceable information; or any other type of unique identifying characteristic, including Web site or IP address, biometric identifiers such as finger or voice print, or photographic image.

Thus, even if the geneticist has eliminated all other specific identifiers, but the label on a sample indicates that the patient is "93 years old" or lives in a certain zip code, the safe harbor will not be met and the information will be HIPAA-protected. Clearly, the most effective and efficient way to de-identify PHI will be to strip it of all unique identifiers, give it an independent office or laboratory number, and provide adequate protection of the re-match key.

\section{Research}

HIPAA also establishes requirements relating to informed consent for the use of PHI for research activities. The threshold question here is whether the health information required for the research is "individually identifiable under" HIPAA. As discussed above, de-identified information is not subject to the Privacy Rules. If the information identifies an individual, or reasonably could be used to identify an individual, it likely will be HIPAA-protected.

The general rule regarding research is that a provider must obtain written authorization to use a patient's PHI for research. The Privacy Rules, however, provide three significant exceptions to this authorization requirement. First, and most importantly, authorization may be waived or altered by an Institutional Review Board or a "privacy board" meeting certain requirements.

Second, an authorization is not necessary if the research will be limited to reviewing information in preparation for research. For example, a geneticist may use PHI without authorization to develop a research protocol, or to assist in the recruitment of research participants. If authorization is waived pursuant to this rationale, the researcher must ensure that no $\mathrm{PHI}$ is removed from the researcher's location during the course of review.

Finally, an authorization is not necessary if the research only uses PHI belonging to deceased persons. Thus, a geneticist will not need to obtain authorization if the research only requires 
information about deceased family members. Although information regarding deceased individuals is protected by HIPAA in other circumstances, it may be used for research purposes without obtaining an authorization.

Even if research does not require an authorization, it is still subject to the minimum necessary standard. Moreover, the research also must comply with other applicable legal requirements. For example, the Privacy Rules regulations do not override the federal Common Rule; where both HIPAA and the Common Rule would apply to research, the provider must comply with both sets of requirements. In addition, the FDA's protection of human subjects regulations - and relevant state law requirements—-must be followed where they are applicable.

\section{PROTECTION OF PATIENTS' RIGHTS}

In addition to the policies and procedures that a health care provider must establish to govern how PHI will be used and disclosed, the Privacy Rules require providers to develop and implement policies and procedures to establish and preserve patients' rights related to their PHI.

First, all providers must maintain a current notice of the provider's privacy practices, including the uses and disclosures of PHI that may be made by the provider, the individual's rights with respect to PHI, and the provider's legal duties. A direct treatment provider must distribute his or her privacy notice to patients when the provider comes in contact with the patient. An indirect treatment provider need not distribute a privacy notice, except at the request of an individual. As with consents, a geneticist based in a hospital or other health care entity may choose to use a privacy notice jointly with the health care entity. As noted above, a joint consent may not be used unless a joint privacy notice also is used.

Second, patients have the right to request additional privacy protections to augment the protections under HIPAA. HIPAA does not require that a geneticist agree to any additional protections. If the geneticist does agree, however, he or she must comply with the additional protections.

Third, patients have the right to inspect or copy their PHI. If a patient requests the opportunity to review his or her file, the provider may deny the patient such opportunity only under very limited circumstances. For example, if a geneticist determines that release of genetic test results to a patient would likely endanger the life or safety of the patient or another person, the geneticist may deny the patient access to the patient's own genetic information, subject to review by another health care professional. Although the rules identify a few other situations in which a geneticist may deny the individual access, in general, a patient should be permitted to inspect or copy his or her own file.

Fourth, individuals have the right to amend inaccurate or incomplete PHI. Finally, individuals have the right to request an accounting of all disclosures (other than disclosures made for treatment purposes) made during the past 6 years. Under this requirement, the geneticist must be able to trace all disclosures he or she has made for research, educational use, pay- ment, and all other nontreatment uses, and provide such information to the patient upon the patient's request.

\section{ADMINISTRATIVE COMPLIANCE}

The Privacy Rules also require health care providers to meet a number of administrative requirements to protect PHI, including (1) designating a privacy official; (2) designating a contact person or office to receive complaints, and establishing a complaint process; (3) training workforce members to ensure that they understand and will comply with the HIPAA privacy rules, and establishing and applying sanctions for noncompliance; (4) establishing procedures to mitigate any harmful effects of improper uses or disclosures; (5) establishing policies to prohibit requiring individuals to waive their rights, and to prohibit retaliatory or intimidating acts against individuals who file a complaint about a violation of the Privacy Rules; and (6) documenting compliance with required policies and procedures and maintaining such documentation for 6 years. $\mathrm{CH} 1$ 2169078v1

\section{ENFORCEMENT AND PENALTIES}

The HHS Office for Civil Rights has enforcement authority for the Privacy Rules. HIPAA provides civil penalties for failure to comply ( $\$ 100$ per violation; up to $\$ 25,000$ per year) and criminal penalties for wrongful disclosure (up to $\$ 250,000$ and/or 10 years in prison for violation with intent to sell or use PHI for commercial advantage, personal gain or malicious harm).

\section{CONCLUSIONS}

What more can I say? As expected, the Privacy Rules generally have received praise from privacy advocates and criticism from the health care industry, which believes them to be overly burdensome and unworkable. Moreover, HIPAA provides only a federal floor of protection. More stringent state medical privacy laws will remain in effect. Furthermore, HHS also has proposed separate HIPAA rules governing the physical security of health information. Thus, while compliance with HIPAA's Privacy Rules are necessary, they may not (believe it or not) be sufficient.

Like many controversial statutes and regulations, the HIPAA Privacy Rules will be reviewed, discussed, debated, and probably litigated. HHS may modify them at any time during the first year of implementation, and up to once each year thereafter. Secretary Thompson already has said that he soon will release "very detailed guidance" and recommend modifications to "ensure the quality of care does not suffer inadvertently from the rule."

Whatever the outcome, it is clear that the focus on medical privacy is here to stay. Hopefully, this will encourage patients to avail themselves of appropriate genetic screening and testing, and to participate in research without fear of loss of their medical privacy. In any case, there is a lot to do under the Privacy Rules; and April 14, 2003, is not really that far away. 